\title{
Assembly of Multilayered Films Using Well-Defined, End-Labeled Poly(acrylic acid): Influence of Molecular Weight on Exponential Growth in a Synthetic Weak Polyelectrolyte System
}

Bin Sun, ${ }^{1}$ Christopher M. Jewell, ${ }^{2}$ Nathaniel J. Fredin, ${ }^{2}$ and David M. Lynn ${ }^{1,2, *}$

${ }^{I}$ Department of Chemistry and ${ }^{2}$ Department of Chemical and Biological Engineering, University of Wisconsin - Madison, 1415 Engineering Drive, Madison, Wisconsin 53706

Supplementary Material

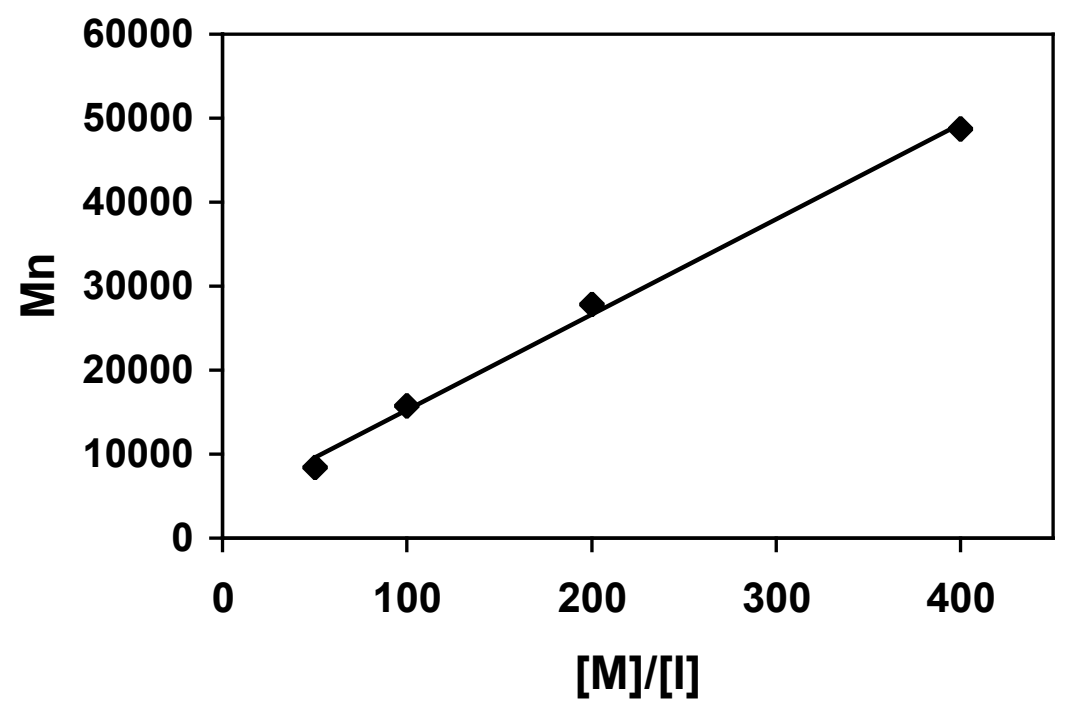

Figure S1: Plot of number average molecular weight $\left(M_{n}\right)$ versus monomer/initiator ratio $([\mathrm{M}] /[\mathrm{I}])$ for the polymerization of polymer $\mathbf{4}$ using initiator $\mathbf{3}$. Molecular weights indicated are relative to monodisperse polystyrene standards. 


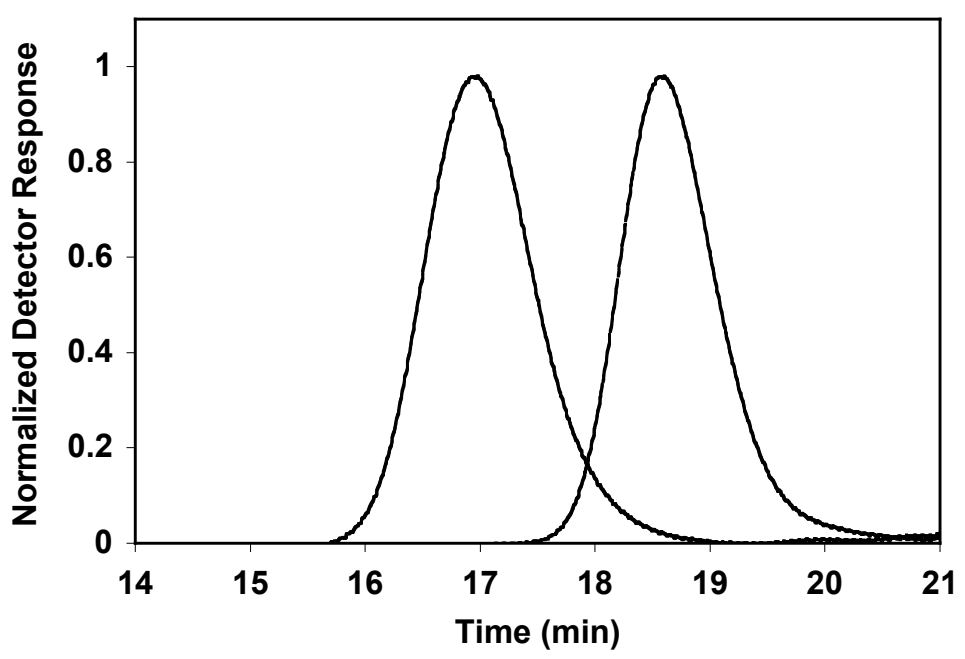

Figure S2: Superimposed gel permeation chromatography (GPC) traces for two different samples of poly( $t$-butyl acrylate) 4 obtained by atom transfer radical polymerization using phthalamide-functionalized initiator $\mathbf{3}$. The peak on the right corresponds to polymer $\mathbf{4 a}$ (see text), synthesized using a monomer/initiator ratio of 50:1 $\left(\mathrm{M}_{\mathrm{n}} \sim 7,000\right.$; PDI =1.36). The peak on the left corresponds to polymer $\mathbf{4 b}$, synthesized using a monomer/initiator ratio of 400:1 $\left(\mathrm{M}_{\mathrm{n}} \sim 49,000\right.$; PDI =1.27). Inspection of these data reveals that these two polymer samples have molecular weight distributions that are substantially non-overlapping.

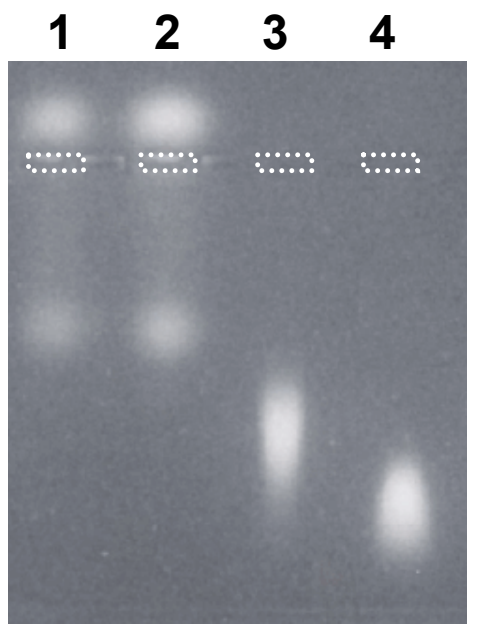

Figure S3: Characterization of fluorescently end-labeled polymers $\mathbf{2 a}$ and $\mathbf{2 b}$ by agarose gel electrophoresis. Lanes labeled 1-4 correspond to 1) TMR-NHS (starting material), 2) TMR-NHS + phthalamide end-labeled polymer $\mathbf{1}, 3)$ polymer $\mathbf{2 b}$, and 4) polymer 2a. Polymer samples (1.0 $\mathrm{mg} / \mathrm{mL}$ in HEPES buffer; $20 \mathrm{mM} ; \mathrm{pH}=7.2$ ) were loaded into wells using a pipette and analyzed on a $2 \%$ agarose gel (HEPES, $20 \mathrm{mM}, \mathrm{pH}=7.2,108 \mathrm{~V}, 1.5 \mathrm{~h}$ ). Fluorescence of TMR was visualized using an ultraviolet lamp. The results shown in lanes 3 and 4, when combined with the control experiments shown in lanes 1 and 2, suggest that the TMR in polymers $\mathbf{2 a}$ and $\mathbf{2} \mathbf{b}$ is covalently linked to these polymers and demonstrate that these samples are free of unreacted TMR. 


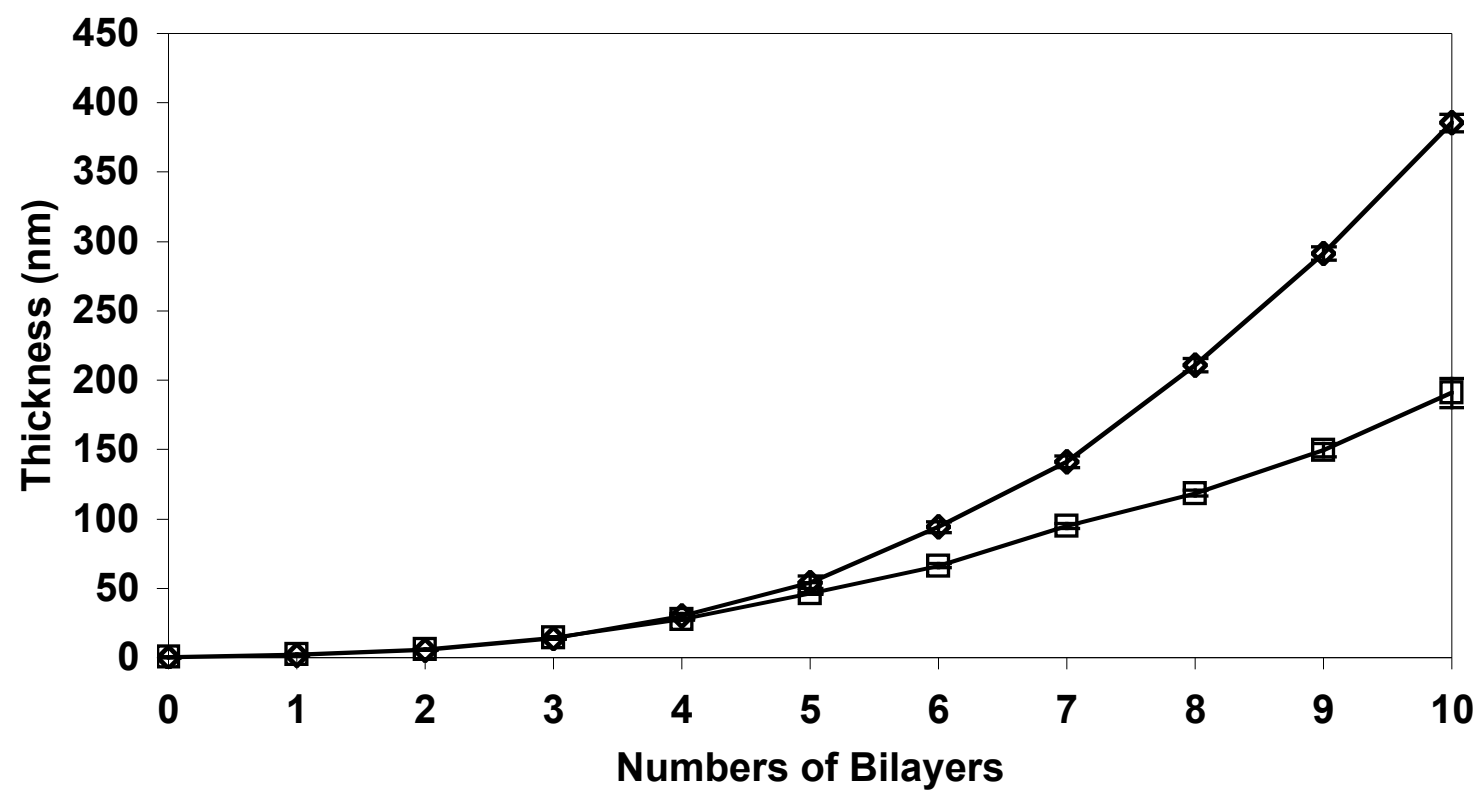

Figure S4: Plot of ellipsometric thickness v. the number of PAH/PAA bilayers deposited for multilayered polyelectrolyte assemblies fabricated using PAH and two different samples of commercially available PAA. Symbols correspond to films fabricated using PAA with $\mathbf{M}_{n}$ $\sim 90,000(\square)$ and $M_{n} \sim 15,000(\diamond)$. Each symbol represents the average value of film thickness calculated from individual measurements of three different films in each case. 